\title{
Multilayered Nanoplasmonic Arrays for Self-Referenced Biosensing
}

\author{
Justin R. Sperling, ${ }^{\circledR}$ Gerard Macias, Steven L. Neale, and Alasdair W. Clark* \\ Biomedical Engineering Research Division, School of Engineering, University of Glasgow, Glasgow G128LT, U.K.
}

\section{Supporting Information}

\begin{abstract}
Nanostructured sensors based on localized surface plasmon resonance (LSPR) offer a number of advantages over other optical sensing technologies, making them excellent candidates for miniaturized, label-free chemical and biological detection. Highly sensitive to local refractive index changes, the resonance peaks of the nanosensors shift by different amounts when subject to different biological and chemical environments. Modifications to the nanostructure surface allow for the detection of specific molecules and chemicals with shifts so sensitive that the presence of single
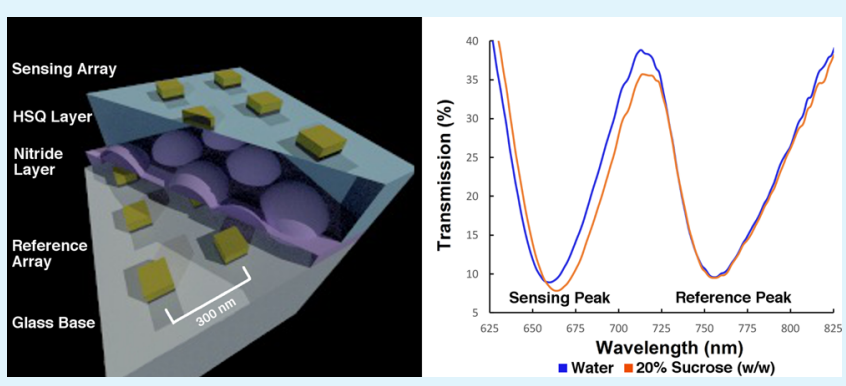
molecules can be detected. However, this extreme sensitivity has its drawbacks. Resonance shifts also occur because of temperature shifts, light-intensity fluctuations, and other environmental factors. To distinguish detection from drift, a secondary sensor region is often required. This often doubles the size of the device, requires two light sources and detectors (or complex optics), doubles the sample volume required (which may be expensive, or may not be possible if the sample quantity is limited), and subjects the reference to potential biofouling. Here, we present a new proof-of-concept multilayered LSPR sensor design that incorporates both a sensing layer and an encapsulated reference layer within the same region. By doing so, we are able to monitor and correct for sensor drift without the need for a secondary reference channel. We demonstrate the suitability of this sensor for sucrose concentration measurements and for the detection of biotin-avidin interactions, while also showing that the sensor can self-correct for drift. We believe that this multilayer sensor design holds promise for point-of-care diagnostics.
\end{abstract}

KEYWORDS: plasmonics, biosensor, LSPR, self-referencing, multilayer

\section{INTRODUCTION}

Surface plasmon resonance (SPR) has long been used as a label-free optical biosensing technique. ${ }^{1}$ Although a powerful tool for sensing, the use of SPR sensors in point-of-care devices is typically hindered by miniaturization problems and complex supporting optics. ${ }^{2,3}$ As an alternative, sensors based on localized SPR (LSPR) have been demonstrated to have performance similar to SPR sensors. ${ }^{4}$ SPR relies on the propagation of surface plasmon polaritons on a continuous metal film, whereas LSPR is localized to the structures on the nanoscale. Therefore, LSPR allows for the use of more compact optics, does not need precise temperature control, and thus has the potential to be miniaturized beyond the capabilities of SPR sensors. ${ }^{5}$ For example, LSPR sensors were demonstrated in the parallel biosensing of 32 independent sensor elements on the same chip. ${ }^{2}$

LSPR sensors have a characteristic spectral response governed by the size, arrangement, shape, and material of nanostructures that make up the sensor. Additionally, the interaction of the nanostructure's surface plasmons with the local environment (about $100 \mathrm{~nm}$ ), external light source, and external temperature also affects the response. Binding events are thus typically measured by detecting a shift in the spectral response from the baseline caused by the interaction of the LSPR with the bound molecule. This highly localized sensing minimizes bulk refractive index (RI) effects ${ }^{3,6,7}$ and has been used for real-time, label-free detection of nanoscale biochemical events such as protein-substrate binding, $5,6,8,9$ antibody-antigen binding, ${ }^{2,3,10}$ and DNA base pairing., ${ }^{31-13}$

As LSPR sensing devices are responsive to many different changes on the nanoscale, a major challenge in real-time detection with these devices is determining if a measured shift in response arises from a desired signal or some sort of drift in the baseline. ${ }^{14}$ This is especially problematic for the devices designed for point-of-care applications, where a sensor is more likely to be exposed to environments where a precise control of external factors-such as intensity fluctuations of the light source, for example-is not possible. To account for such drifts, a reference for the sensing device is necessary. The standard method to account for the baseline drift from the environment is to use a separate reference flow channel. ${ }^{15,16}$ However, this approach neglects the possibility that the events in two separate channels may differ.

To overcome this challenge, a variety of sensor technologies with the capability to self-reference have been explored. Examples include dual-metal SPR sensors, ${ }^{17,18}$ modified Mach-Zehnder interferometer phase sensors, ${ }^{19-21}$ porous silicon double-layer sensors, ${ }^{22,23}$ photochemical sensors, ${ }^{24}$

Received: July 25,2018

Accepted: September 12, 2018

Published: September 12, 2018 

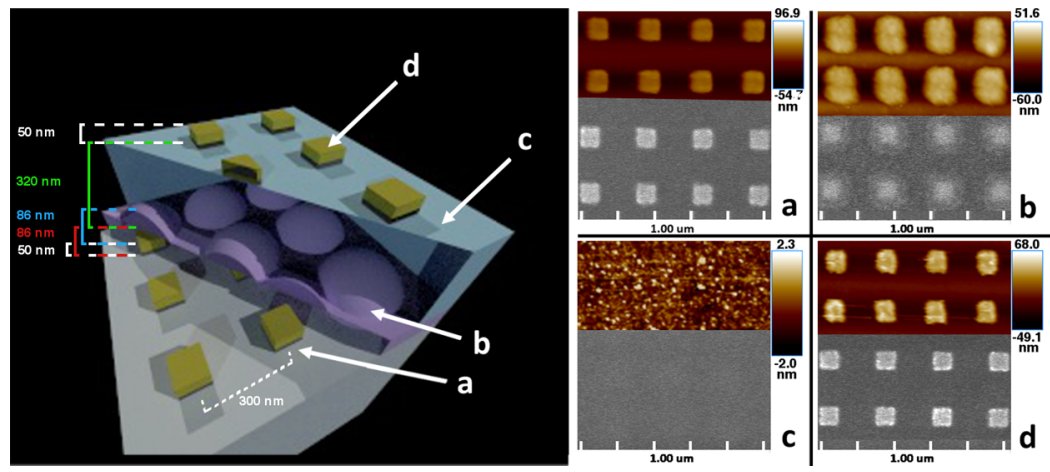

Figure 1. Schematic and SEM/AFM images of a multilayered sensing device. Left: Schematic showing the multilayered device. (a) Gold nanostructure LSPR reference layer encapsulated in (b) a conforming silicon nitride with (c) an HSQ planarization layer and (d) a gold nanostructure LSPR sensing layer. The nanostructures are $100 \times 100 \times 50 \mathrm{~nm}(L \times W \times H)$. Right: AFM (top) and SEM (bottom) images of each of the device layers $(\mathrm{a}-\mathrm{d})$ taken at the time of deposition. The AFM images confirm that the HSQ layer planarized the nitride layer.

nanohole LSPR sensors, ${ }^{25}$ refractometric LSPR sensors, ${ }^{26}$ dualmetal suspended colloid LSPR sensors, ${ }^{27}$ and polarizationdependent sensors, ${ }^{14}$ to name a few.

Here, we present a multilayered LSPR sensor with internal referencing that self-corrects for baseline drift. Composed of both an encapsulated and an exposed nanoplasmonic layer, this device exhibits two distinct peaks: a reference peak from the encapsulated layer and a sensing peak from the exposed layer. The presence of the reference layer directly below the sensing layer allows us to correct for any drift that may occur during real-time chemical and biosensing experiments using a single measurement from a single beam of light.

Additionally, utilizing one flow channel rather than separate sensing and referencing channels allows for a more compact design and eliminates the need to inject potentially expensive samples into a parallel reference channel. ${ }^{17}$

Our design offers advantages over other technologies in that our self-referencing mechanism does not require a spatial control over different surface modifications ${ }^{17,18}$ and is not subject to fouling because the reference layer is isolated from the sensing region. ${ }^{17,18,22-26}$ Additionally, our device selfcorrects rather than just recognizing "unreliable data," ${ }^{24}$ can be regenerated and thus reused in multiple detection events rather than being consumed during detection, ${ }^{27}$ and does not require multiple detectors ${ }^{26}$ or a beam-splitter. ${ }^{14}$

\section{EXPERIMENTAL SECTION}

Device Fabrication. The devices were fabricated using electronbeam lithography, inductively coupled plasmon chemical vapor deposition (ICP-CVD), and metal evaporation. For both the reference and sensing nanoantenna layers, a resist bilayer of $4 \%$ poly(methyl methacrylate) (PMMA) 2010 and 2.5\% PMMA 2041 (total thickness $150 \mathrm{~nm}$ ) was patterned using a Vistec VB6 ultrahigh resolution extra-wide field electron-beam lithography tool. Following the development of the pattern, a $2 / 50 \mathrm{~nm} \mathrm{Ti} / \mathrm{Au}$ layer was evaporated onto the sample using a Plassys MEB 400S/550S electronbeam evaporation tool. The silicon nitride layer was deposited using an Oxford Instruments System 100 ICP 180 PECVD nitride deposition tool at room temperature. A J.A. Woollam Mark II variable angle spectroscopic ellipsometer was used to determine the thickness and dielectric $(n, k)$ values of the nitride. To planarize the nitride layer, hydrogen silsesquioxane (HSQ, Fox 16) was spun on and then cross-linked using the electron-beam lithography tool. The final sensing nanoantenna layer was then fabricated on top of the HSQ layer, following the same procedure as the fabrication steps of the reference layer.
Experimental Setup. A polydimethylsiloxane (PDMS) microfluidic channel was placed on top of the device, and a positive crankcase ventilation value was used to allow for sample injection without disrupting the flow. The flow rate from the microfluidic pump was set to $100 \mu \mathrm{L} \cdot \mathrm{min}^{-1}$. A custom-built microspectrophotometer was used to measure the real-time transmission spectra $(0.5 \mathrm{~nm}$ resolution). Light from a visible to near-infrared (VIS-NIR) light source (tungsten-halogen $400-1200 \mathrm{~nm}$ wavelength) was used to probe the sensor. A $10 \times$ objective was used to couple the transmitted light into an optical fiber attached to a StellarNet Microspectrophotometer (StellarNet Blue Wave). Sample rates of $7.5 \mathrm{~s}$ for the RI calibration experiment and $3.5 \mathrm{~s}$ for the biotin-avidin experiment were used to capture a 30-measure-averaged transmission response.

Peak Analysis. MatLab and Origin were used to analyze the transmission spectra for each series of the measurements. The transmission spectrum was smoothed (20 points, mean average smoothing) and interpolated (from 0.5 to $0.01 \mathrm{~nm}$ ). The peak position values of the two minima peaks and the full width at halfmaximum (fwhm) values were determined. The corrected position response $(\mathrm{CPR})$ was calculated by subtracting the position of the sensing peak from the reference peak.

Sensor Calibration. For the calibration experiments, water containing varying concentrations of dissolved sucrose (SigmaAldrich), from 0.5 to $50 \%(\mathrm{w} / \mathrm{w})$, was introduced to the device. The RI of the surrounding medium was, thus, altered from 1.333 to $1.4201 .^{28}$

Device Functionalization. The sensor was immersed in a $10 \mathrm{mM}$ ethanolic solution of 11-mercaptoundecanoic acid (MUA, Sigma Aldrich) overnight, rinsed three times with ethanol, dried under a stream of nitrogen, and assembled with the microfluidic system. A constant flow rate of $100 \mu \mathrm{L} \cdot \mathrm{min}^{-1}$ was maintained throughout the experiments. The channel was flushed with water for $20 \mathrm{~min}$ prior to the injection of $500 \mu \mathrm{L}$ of $200 \mathrm{mM}$ 1-ethyl-3-(3- dimethylaminopropyl)-carbodiimide (Sigma-Aldrich) in MES buffer at $\mathrm{pH} 5.5$ and rinsed with deionized (DI) water for $15 \mathrm{~min} .500 \mu \mathrm{L}$ of $500 \mu \mathrm{g} \cdot \mathrm{mL}^{-1}$ biotin hydrazide (Sigma-Aldrich) in DI water was then injected, followed by a DI water rinse for a further $15 \mathrm{~min}$. Finally, the unreacted sites were blocked with $500 \mu \mathrm{L}$ of $1 \mathrm{M}$ ethanolamine (Sigma-Aldrich) in DI water and rinsed with DI water for $15 \mathrm{~min}$.

Biological Sensing. Throughout the experiments, $0.1 \%$ Tween20 in phosphate-buffered saline (PBS) was used as the flowing solution. Prior to biological sensing, the device was stabilized by flowing the PBS solution at $100 \mu \mathrm{L} \cdot \mathrm{min}^{-1}$ for $30 \mathrm{~min}$. Throughout the experiments, the flow rate was kept constant at $100 \mu \mathrm{L} \cdot \mathrm{min}^{-1}$, and at least $10 \mathrm{~min}$ of baseline were acquired before biomolecule injection. Sensor regeneration was achieved by injecting $500 \mu \mathrm{L}$ of $20 \mathrm{mM}$ sodium hydroxide in DI water. As a nonspecific control, $500 \mu \mathrm{L}$ of $100 \mu \mathrm{g} \cdot \mathrm{mL}^{-1}$ bovine serum albumin (BSA) was injected. Finally, 0.1, 1,100 and $500 \mu \mathrm{g} \cdot \mathrm{mL}^{-1}$ of avidin in $0.1 \%$ Tween-20 PBS was injected 
at a constant volume of $500 \mu \mathrm{L}$ to test the specificity and dose dependency of our device.

\section{RESULTS/DISCUSSION}

The multilayered substrate design (Figure 1) consists of two $100 \mathrm{~nm} \times 100 \mathrm{~nm} \times 50 \mathrm{~nm}(L \times W \times H)$ gold nanoarrays-an encapsulated reference array (Figure 1a) and an exposed sensing array (Figure 1d). By encapsulating the reference array in silicon nitride (Figure 1b), its LSPR is isolated from the environment of the exposed sensing array. ${ }^{6}$ Nanosquare shapes were chosen for this proof-of-concept device because (1) the design allows for easy, repeatable fabrication, (2) the symmetrical shape is not subject to a polarization-dependent response, and (3) the shape maximizes the surface area coverage for a given periodicity (thus maximizing the plasmonic signal). A periodicity of $300 \mathrm{~nm}$ was chosen to provide a significant enough distance between nanostructures to make plasmon coupling negligible, ${ }^{29}$ while still providing a strong plasmonic signal ( $>90 \%$ transmission-attenuation).

An examination of the insulating layers was conducted (see the Supporting Information) to determine (1) the minimum thickness of silicon nitride necessary to insulate the reference layer from the environment above and (2) the minimum height of HSQ needed to planarize the nitride-capped layer (for subsequent fabrication of the sensing layer). It was determined that a distance of $100 \mathrm{~nm}$ was sufficient to isolate the reference layer's nanostructures from the sensing layer's nanostructures and their local environment (Figure S2 and Figure S3). Our chosen nanostructure dimensions provided the largest signal attenuation of all the structures that were trialed, and this was the case for all thicknesses of nitride that were studied (see Supporting Information). It was also determined that an HSQ thickness of $200 \mathrm{~nm}$ was required to planarize the device following nitride deposition (Figure S5). Based on these findings, the thickness of silicon nitride and HSQ used for the final device was $85 \mathrm{~nm}(86.59 \pm 0.042$ $\mathrm{nm}$ as measured by ellipsometry) and $320 \mathrm{~nm}$, respectively; ensuring a planar fabrication base for the sensing layer and complete layer-to-layer plasmonic isolation.

The double plasmonic layer design results in two peaks in the transmission response, denoted as $\mathrm{P}_{1}$ and $\mathrm{P}_{2}$ in Figure 2, corresponding to the sensing peak and reference peak, respectively. To correct for the drift in peak position, the CPR was calculated by subtracting the position of the sensing peak from that of the reference peak

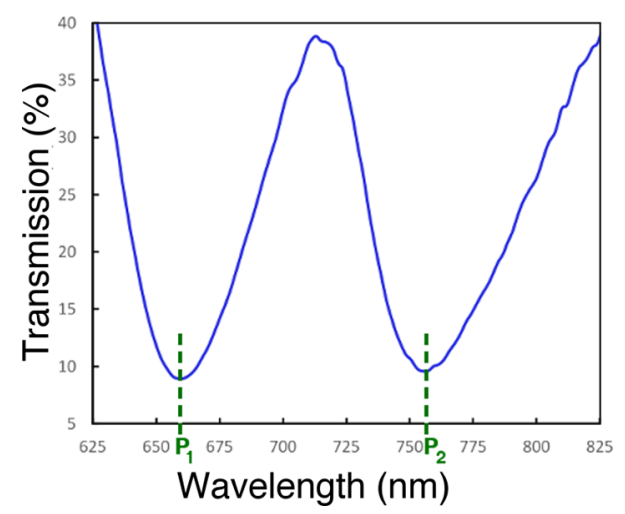

Figure 2. CPR, defined as the difference between the sensing peak $\left(\mathrm{P}_{1}\right)$ and the encapsulated reference peak $\left(\mathrm{P}_{2}\right)$.

$$
\operatorname{CPR}[\mathrm{nm}]=\mathrm{P}_{2}-\mathrm{P}_{1}
$$

To measure the spectral response of the sensor, a custommade microspectrophotometer was used to measure the transmission response caused by fluid flow through a PDMS microfluidic channel placed on top of the device. The stability of the reference peak is demonstrated in Figure 3a,b. When the

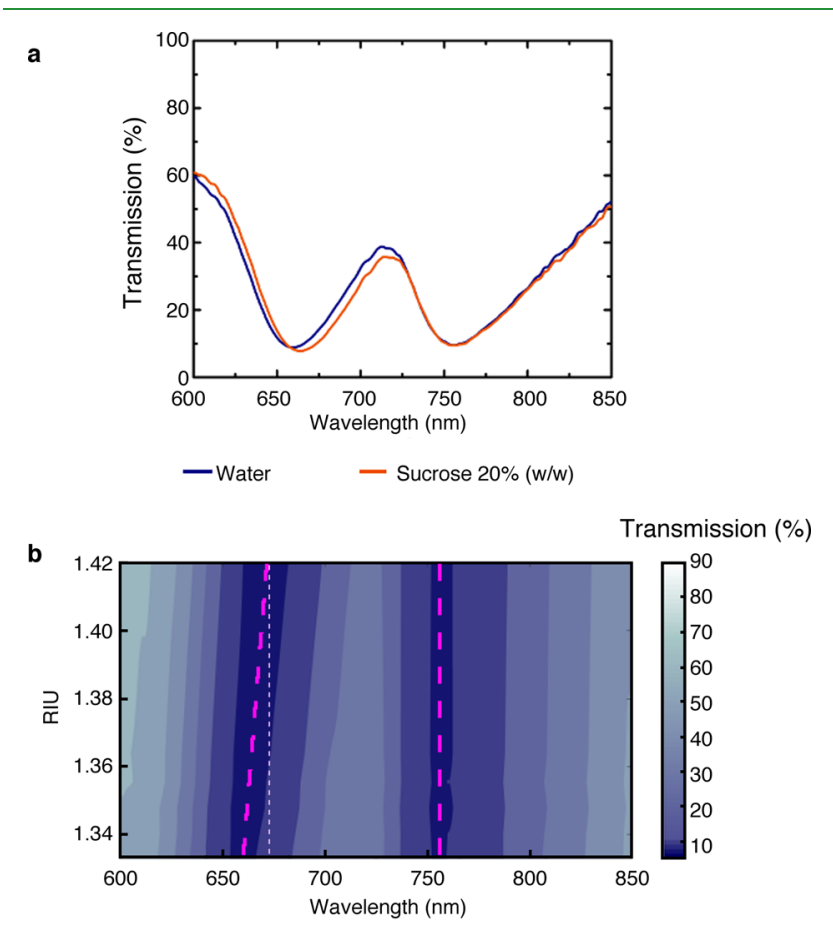

c

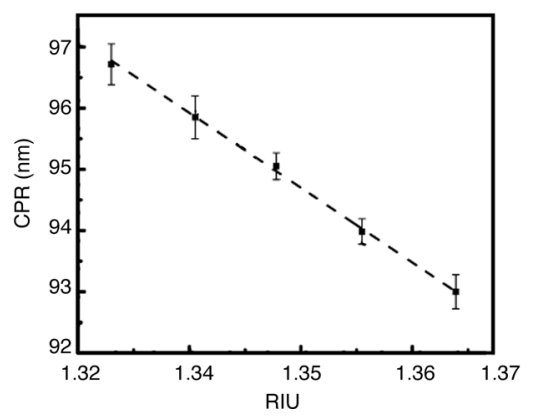

Figure 3. Experimental data from the multilayered device. (a) Transmission plot of the device in response to water $(\mathrm{RIU}=1.333)$ and $20 \%$ sucrose $(\mathrm{w} / \mathrm{w})(\mathrm{RIU}=1.3639)$. (b) Contour plot of the transmission of multilayered sensor in $0,5,10,15,20$, and $50 \%(\mathrm{w} / \mathrm{w})$ sucrose solutions. Significantly, the encapsulated reference peak (rightmost dashed line) does not exhibit a shift in response with varying RIs, whereas the uncapped sensing peak (leftmost dashed line) does. (c) Experimental results of CPR to changes in RI. The experimental CPR has a sensitivity of $122 \pm 3 \mathrm{~nm} \cdot \mathrm{RIU}^{-1}$. The CPR curve is best described with a linear fit because it has an adjusted $R^{2}$ value well above $99 \%$.

RI surrounding the sensor is changed from that of water $(\mathrm{RI}=$ $1.333)$ to that of $20 \%(\mathrm{w} / \mathrm{w})$ sucrose solution $(\mathrm{RI}=1.3639)$, a clear shift in $\mathrm{P}_{1}$ occurs, whereas $\mathrm{P}_{2}$ remains constant (Figure $3 a$ ). Figure $3 b$ expands upon this, showing a contour plot of the transmission of the sensor with varying RIs of 1.333, $1.3405,1.3478,1.3555,1.3639$, and 1.4201 . The reference peak (rightmost dashed line) does not exhibit a shift in response with varying RIvalues, whereas the sensing peak 
a(i)

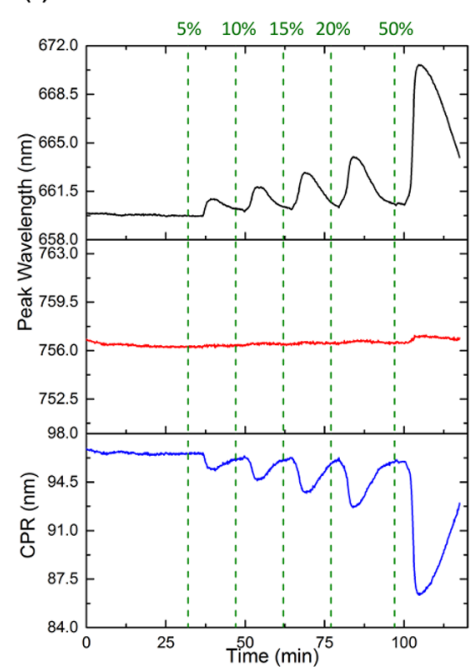

a(ii)

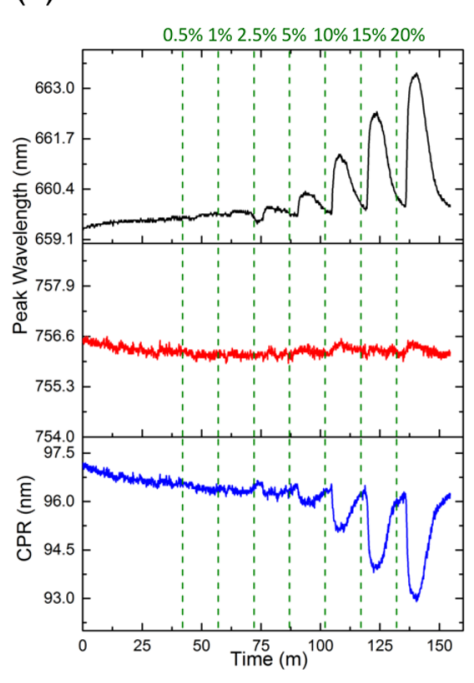

a(iii)

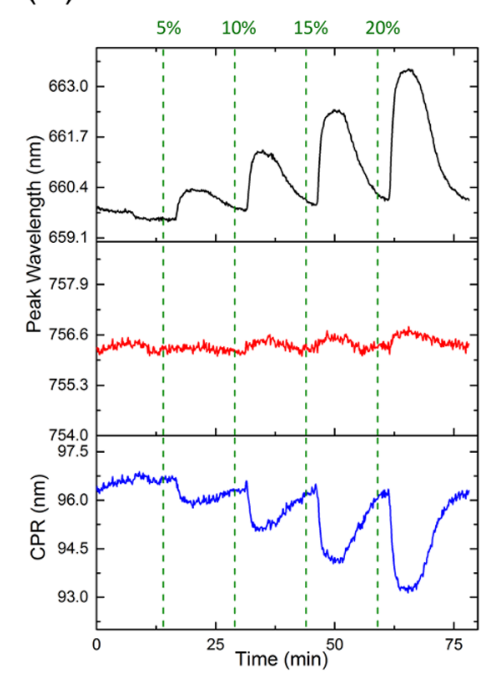

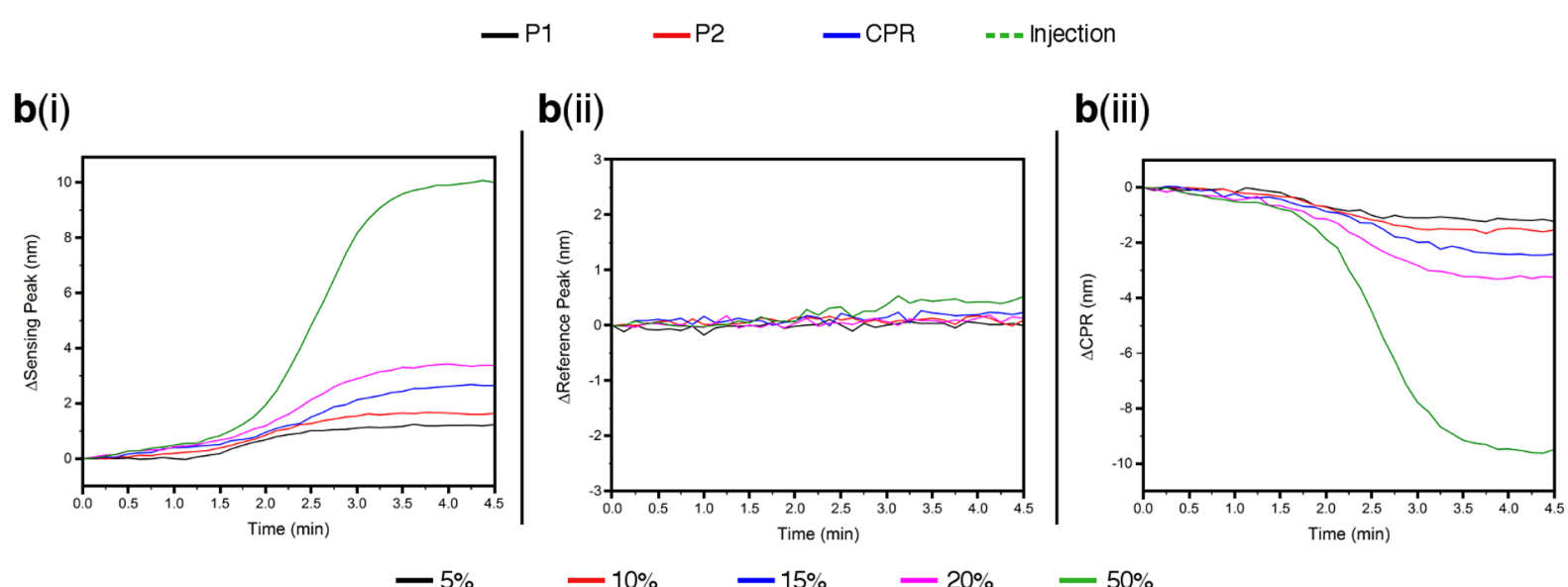

Figure 4. Real-time response of sensor to changes in RI. (a) Real-time response of the sensing layer P1 (black), reference layer P2 (red), and calculated CPR (blue) to varying RI changes using sucrose solutions (w/w in water) of (i) $0,5,10,15,20$, and $50 \%$; (ii) $0,0.5,1.0,2.5,5,10,15$, and $20 \%$; and (iii) $0,5,10,15$, and $20 \%$. The dotted green lines indicate the time at which sucrose was injected into the system. For each run, during the first $10 \mathrm{~min}$, the device is stabilizing at baseline. The measurements in [a(ii,iii)] were taken in succession of the previous set of measurements. In the first $10 \mathrm{~min}$ of the measurement, the sensor was returning to baseline from the sucrose solution of the previous run. (b) The shift in peak for (i) sensing layer, (ii) reference layer, and (iii) calculated CPR for the five injections from [a(i)].

(leftmost dashed line) does. This indicates that the encapsulated peak is a viable reference, independent from the RI changes of the sensing region. The reference peak can, therefore, be used to correct the position the sensing layer's peak.

The sensitivity of the sensor was determined from the slope of the CPR-RI curve (Figure 3c). The CPR sensitivity of 122 $\pm 3 \mathrm{~nm} \cdot \mathrm{RIU}^{-1}$ and the figure-of-merit $(\mathrm{FoM})^{30}$ of 1.3 make it close to the sensitivity shown for silver nanocubes, ${ }^{31}$ but overall, at the lower sensitivity range of other LSPR sensing devices, which range from 90 to $1000 \mathrm{~nm} \cdot \mathrm{RIU}^{-1}$ and FoM of 0.8-5.4..$^{3,5-7}$ As a proof of concept, this design shows the potential as a new means to self-reference. It is postulated that utilizing this multilayer design with plasmonic structures known to be more sensitive, such as a dimer design, ${ }^{26}$ will improve the sensitivity.

To further demonstrate the stability, real-time measurements of induced RI changes were performed. Figure 4a shows the real-time position response of the sensing peak (black) and the reference peak (red), as well as the self-corrected response (blue) of the sensor to injections of sucrose solutions in water with varying RIs. The injections (in percent weight by weight) for the runs were (i) $0,5,10,15,20$, and $50 \%$, (ii) $0,0.5,1.0$, $2.5,5,10,15$, and $20 \%$, and (iii) $0,5,10,15$, and $20 \%$. For each run, the device was left to stabilize at baseline, and the injections of sucrose were only started following $10 \mathrm{~min}$ of stabilized response. During the first $20 \mathrm{~min}$, the device was stabilizing at baseline. The measurements in Figure 4aii,iii were taken in quick succession. As a result, for the first $10 \mathrm{~min}$ of these two runs, the sensor was still returning to baseline from the last injection of sucrose from the previous run. Figure $4 b$ shows the shift in (i) the sensing layer, (ii) the reference layer, and (iii) the calculated CPR from the baseline measurements for the five injections shown in Figure 4ai. Both the sensing peak position and CPR show a stepwise change in response when higher concentrations of sucrose-water are injected in the system, whereas the reference does not. As the measurements of the second run (Figure 4aii) have the most fluctuation, this run was used to determine the noise, limit of detection, and limit of quantification of the sensor. Using the full $10 \mathrm{~min}$ prior to the first injection of run 2 as the baseline, the standard deviation of the baseline (noise), limit of 
detection $(2 \times$ noise $)$, and limit of quantification ( $3 \times$ noise) were determined from $C P R .{ }^{32}$ The sensitivity for CPR was then used to calculate the corresponding change in RI. The limits of detection and quantification for the sensor by CPR are the changes of $1.52 \times 10^{-3}$ and $2.28 \times 10^{-3}$ RIU, respectively (see Table 1). Although these values are on the lower end of

Table 1. Noise Level, Limit of Detection, and Limit of Quantification for CPR and the Corresponding RI Values

$\begin{array}{lcc} & \Delta \mathrm{CPR} & \Delta \mathrm{RIU}(\mathrm{CPR}) \\ \text { noise level } & 9.28 \times 10^{-2} & 7.59 \times 10^{-4} \\ \text { limit of detection } & 1.86 \times 10^{-2} & 1.52 \times 10^{-3} \\ \text { limit of quantification } & 2.78 \times 10^{-2} & 2.28 \times 10^{-3}\end{array}$

other published LSPR sensor devices, ${ }^{6,7,33}$ we have successfully demonstrated a new design for self-referencing devices that can now be modified with much more sensitive nanostructure arrays.

To demonstrate the potential of the self-correcting device for biosensing applications, we performed a biochemical binding assay. For this purpose, we chose the well-known biotin-avidin interaction as a model. Prior to the real-time measurements, the nanoplasmonic device was functionalized with MUA. ${ }^{33,34}$ This treatment provided a self-assembled monolayer (SAM) with carboxylic acid moieties that were further used to covalently attach biotin hydrazide via ethylcarbodiimide cross-linking. ${ }^{34}$ The unreacted sites on the nanostructures were blocked with ethanolamine, ${ }^{33}$ and Tween20 was added to the PBS solution used during the biosensing experiment to prevent avidin from binding to the unblocked regions of the glass base of the device.

Figure 5a shows the change in CPR to an injection of 500 $\mu \mathrm{g} \cdot \mathrm{mL}^{-1}$ avidin (green dotted line). To regenerate the surface after protein binding, $20 \mathrm{mM} \mathrm{NaOH}$ was injected into the device (purple dotted line). To verify the suitability of the sensor in providing a dose-dependent response, three additional concentrations of avidin $\left(0.1,1\right.$, and $\left.100 \mu \mathrm{g} \cdot \mathrm{mL}^{-1}\right)$ were injected. Additionally, the specificity of the sensor was tested by injecting BSA at a concentration of $100 \mu \mathrm{g} \cdot \mathrm{mL}^{-1}$. Figure $5 \mathrm{~b}$ shows the CPR response to BSA and all four concentrations of avidin. CPR is able to detect avidin in a dose-dependent manner. We also observed that the injection of $100 \mu \mathrm{g} \cdot \mathrm{mL}^{-1}$ of BSA did not produce a significant shift in the response of our sensor, thus demonstrating the specificity to avidin. However, the concentrations of avidin where a significant shift could be observed were 100 and $500 \mu \mathrm{g} \cdot \mathrm{mL}^{-1}$. The sensitivity may be improved by investigating different antenna geometries.

\section{CONCLUSIONS}

In summary, we presented a proof-of-concept multilayered LSPR device with an encapsulated reference region that exhibits drift self-correction. We successfully demonstrate the use of the device as a biosensor to detect selectively and in a dose-dependent manner the biotin-avidin interaction. We believe that combining the concept of an encapsulated referencing layer with different LSPR structures may improve the sensitivity. The inclusion of both the reference and sensing regions within the same physical space allows for a more compact design, eliminates the need to inject samples that are potentially expensive or of limited quantity into a noncapturing parallel reference channel, and allows for the use of a single detector rather than multiple detectors. This is especially of interest for point-of-care diagnostic devices because the selfreferencing design accounts for limitations in resources and sample quantity, operates in transmission mode (standard $\mathrm{UV}$-vis instrumentation at point of care is generally carried out in this mode), ${ }^{5}$ and can be incorporated into multiplexed microfluidic systems.

\section{ASSOCIATED CONTENT}

\section{S Supporting Information}

The Supporting Information is available free of charge on the ACS Publications website at DOI: 10.1021/acsami.8b12604.

Simulations to determine minimum nitride thickness and minimum vertical distance between nanostructure array layers, experimental determination of minimum HSQ layer thickness, full device simulations, and measurements of RI values of the nitride layer (PDF)

\section{AUTHOR INFORMATION}

\section{Corresponding Author}

*E-mail: alasdair.clark@glasgow.ac.uk. ORCID

Justin R. Sperling: 0000-0002-1708-2474
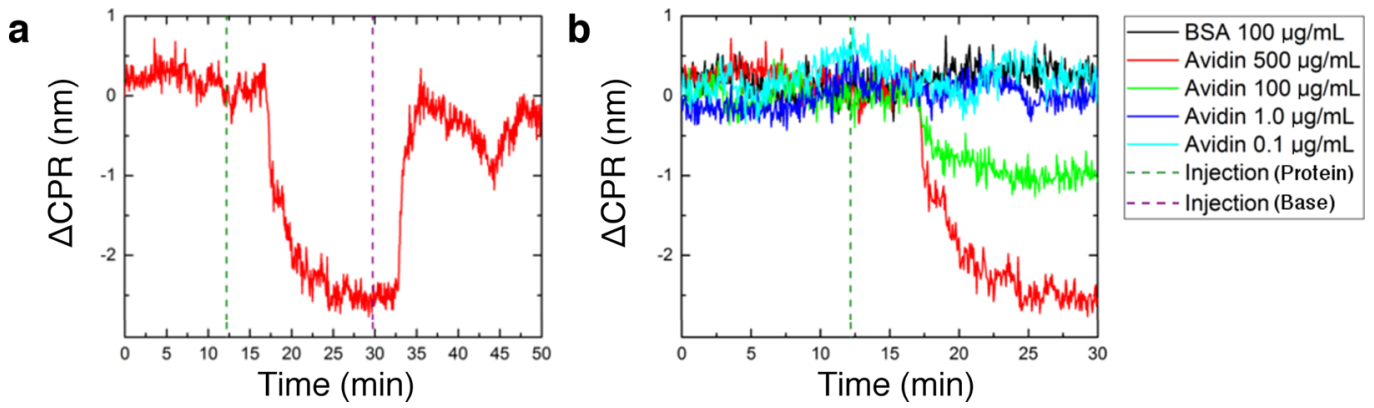

Figure 5. Real-time biosensing of the biotin-avidin interaction. (a) The real-time change of CPR to the specific binding and $\mathrm{pH}$-mediated release of $500 \mu \mathrm{g} \cdot \mathrm{mL}^{-1}$ avidin to biotin attached to the gold nanostructures on the surface of the sensing region of the device. The dotted green line denotes avidin injection. The dotted purple line denotes when $20 \mathrm{mM} \mathrm{NaOH}$ was injected to mediate the release of the protein from the surface. The fluctuation after $\mathrm{NaOH}$ injection is present because of the change of the $\mathrm{RI}$ of solutions flowing through the device. Prior to injecting any other concentrations of protein into the device, a long flush with PBS was done to return the signal to baseline. (b) The real-time change of CPR to the nonspecific interaction of BSA $\left(100 \mu \mathrm{g} \cdot \mathrm{mL}^{-1}\right)$ and the specific binding of avidin $\left(0.1,1,100\right.$, and $\left.500 \mu \mathrm{g} \cdot \mathrm{mL}^{-1}\right)$ with biotin. The dotted line in the plot denotes the time at which the protein was injected into the system. 


\section{Author Contributions}

The authors contributed equally. All authors conceived and designed the experiments. All authors contributed to the writing and editing of the manuscript. J.R.S. and G.M. simulated, designed, and fabricated the devices, performed the experiments, and evaluated the data. J.R.S. and G.M. contributed equally to the work. A.W.C. and S.L.N. coordinated and supervised the work. All authors have given approval to the final version of the manuscript.

\section{Notes}

The authors declare no competing financial interest.

\section{ACKNOWLEDGMENTS}

This work was supported by The Leverhulme Trust (grant RPG-2014-343), EPSRC (grant EP/P51133X/1) and BBSRC (grant BB/N016734/1). The authors also wish to thank all the staff working in the James Watt Nanofabrication Centre for their support.

\section{ABBREVIATIONS}

AFM, atomic force microscopy; BSA, bovine serum albumin; $\mathrm{CPR}$, corrected position response; DI, deionized (water); DNA, deoxyribonucleic acid; FoM, figure of merit; fwhm, full width half-maximum; HSQ hydrogen silsesquioxane; LSPR, localized surface plasmon resonance; MES, 2-( $N$-morpholino)ethanesulfonic acid; MUA, 11-mercaptoundecanoic acid; NIR, near-infrared light; PBS, phosphate-buffered saline; PDMS, polydimethylsiloxane; PMMA, poly(methyl methacrylate); RI, refractive index; RIU, refractive index unit; SAM, selfassembled monolayer; SEM, scanning electron microscopy; SPR, surface plasmon resonance; UV, ultraviolet light; VIS, visible light

\section{REFERENCES}

(1) Homola, J. Surface Plasmon Resonance Sensors for Detection of Chemical and Biological Species. Chem. Rev. 2008, 108, 462-493.

(2) Aćimović, S. S.; Ortega, M. A.; Sanz, V.; Berthelot, J.; GarciaCordero, J. L.; Renger, J.; Maerkl, S. J.; Kreuzer, M. P.; Quidant, R. Lspr Chip for Parallel, Rapid, and Sensitive Detection of Cancer Markers in Serum. Nano Lett. 2014, 14, 2636-2641.

(3) Aćimović, S. S.; Ś́pová, H.; Emilsson, G.; Dahlin, A. B.; Antosiewicz, T. J.; Käll, M. Superior Lspr Substrates Based on Electromagnetic Decoupling for on-a-Chip High-Throughput LabelFree Biosensing. Light: Sci. Appl. 2017, 6, e17042.

(4) Svedendahl, M.; Chen, S.; Dmitriev, A.; Käll, M. Refractometric Sensing Using Propagating Versus Localized Surface Plasmons: A Direct Comparison. Nano Lett. 2009, 9, 4428-4433.

(5) Unser, S.; Bruzas, I.; He, J.; Sagle, L. Localized Surface Plasmon Resonance Biosensing: Current Challenges and Approaches. Sensors 2015, 15, 15684-15716.

(6) Mayer, K. M.; Hafner, J. H. Localized Surface Plasmon Resonance Sensors. Chem. Rev. 2011, 111, 3828-3857.

(7) Szunerits, S.; Boukherroub, R. Sensing Using Localised Surface Plasmon Resonance Sensors. Chem. Commun. 2012, 48, 8999-9010.

(8) Langer, J.; Novikov, S. M.; Liz-Marzan, L. M. Sensing Using Plasmonic Nanostructures and Nanoparticles. Nanotechnology 2015, 26, 322001 .

(9) Sagle, L. B.; Ruvuna, L. K.; Ruemmele, J. A.; Van Duyne, R. P. Advances in Localized Surface Plasmon Resonance Spectroscopy Biosensing. Nanomedicine 2011, 6, 1447-1462.

(10) Fernandez, F.; Garcia-Lopez, O.; Tellechea, E.; Asensio, A. C.; Cornago, I. Lspr Cuvette for Real-Time Biosensing by Using a Common Spectrophotometer. IEEE Sens. J. 2016, 16, 4158-4165.
(11) Sönnichsen, C.; Reinhard, B. M.; Liphardt, J.; Alivisatos, A. P. A Molecular Ruler Based on Plasmon Coupling of Single Gold and Silver Nanoparticles. Nat. Biotechnol. 2005, 23, 741-745.

(12) Yoo, S. Y.; Kim, D.-K.; Park, T. J.; Kim, E. K.; Tamiya, E.; Lee, S. Y. Detection of the Most Common Corneal Dystrophies Caused byBIGH3Gene Point Mutations Using a Multispot Gold-Capped Nanoparticle Array Chip. Anal. Chem. 2010, 82, 1349-1357.

(13) Verdoold, R.; Gill, R.; Ungureanu, F.; Molenaar, R.; Kooyman, R. P. H. Femtomolar DNA Detection by Parallel Colorimetric Darkfield Microscopy of Functionalized Gold Nanoparticles. Biosens. Bioelectron. 2011, 27, 77-81.

(14) Wadell, C.; Langhammer, C. Drift-Corrected Nanoplasmonic Hydrogen Sensing by Polarization. Nanoscale 2015, 7, 10963-10969.

(15) Liu, Y.; Liu, Q.; Chen, S.; Cheng, F.; Wang, H.; Peng, W. Surface Plasmon Resonance Biosensor Based on Smart Phone Platforms. Sci. Rep. 2015, 5, 12864.

(16) Nenninger, G. G.; Clendenning, J. B.; Furlong, C. E.; Yee, S. S. Reference-Compensated Biosensing Using a Dual-Channel Surface Plasmon Resonance Sensor System Based on a Planar Lightpipe Configuration. Sens. Actuators, B 1998, 51, 38-45.

(17) Lu, H. B.; Homola, J.; Campbell, C. T.; Nenninger, G. G.; Yee, S. S.; Ratner, B. D. Protein Contact Printing for a Surface Plasmon Resonance Biosensor with on-Chip Referencing. Sens. Actuators, B 2001, 74, 91-99.

(18) Boozer, C.; Yu, Q.; Chen, S.; Lee, C.-Y.; Homola, J.; Yee, S. S.; Jiang, S. Surface Functionalization for Self-Referencing Surface Plasmon Resonance (Spr) Biosensors by Multi-Step Self-Assembly. Sens. Actuators, B 2003, 90, 22-30.

(19) Wu, S. Y.; Ho, H. P.; Law, W. C.; Lin, C.; Kong, S. K. Highly Sensitive Differential Phase-Sensitive Surface Plasmon Resonance Biosensor Based on the Mach-Zehnder Configuration. Opt. Lett. 2004, 29, 2378-2380.

(20) Levy, R.; Ruschin, S. Design of a Single-Channel Modal Interferometer Waveguide Sensor. IEEE Sens. J. 2009, 9, 146-153.

(21) Kashif, M.; Bakar, A. A. A.; Hashim, F. H. Analysing Surface Plasmon Resonance Phase Sensor Based on Mach-Zehnder Interferometer Technique Using Glycerin. Opt. Commun. 2016, $380,419-424$

(22) Pacholski, C.; Sartor, M.; Sailor, M. J.; Cunin, F.; Miskelly, G. M. Biosensing Using Porous Silicon Double-Layer Interferometers: Reflective Interferometric Fourier Transform Spectroscopy. J. Am. Chem. Soc. 2005, 127, 11636-11645.

(23) Pacholski, C.; Yu, C.; Miskelly, G. M.; Godin, D.; Sailor, M. J. Reflective Interferometric Fourier Transform Spectroscopy: A SelfCompensating Label-Free Immunosensor Using Double-Layers of Porous Sio2. J. Am. Chem. Soc. 2006, 128, 4250-4252.

(24) Hao, N.; Zhang, Y.; Zhong, H.; Zhou, Z.; Hua, R.; Qian, J.; Liu, Q.; Li, H.; Wang, K. Design of a Dual Channel Self-Reference Photoelectrochemical Biosensor. Anal. Chem. 2017, 89, 1013310136.

(25) Wang, X.; Chang, T.-W.; Lin, G.; Gartia, M. R.; Liu, G. L. SelfReferenced Smartphone-Based Nanoplasmonic Imaging Platform for Colorimetric Biochemical Sensing. Anal. Chem. 2017, 89, 611-615.

(26) Wersäll, M.; Verre, R.; Svedendahl, M.; Johansson, P.; Käll, M.; Shegai, T. Directional Nanoplasmonic Antennas for Self-Referenced Refractometric Molecular Analysis. J. Phys. Chem. C 2014, 118, 21075-21080.

(27) Rivero, P. J.; Ibañez, E.; Goicoechea, J.; Urrutia, A.; Matias, I. R.; Arregui, F. J. A Self-Referenced Optical Colorimetric Sensor Based on Silver and Gold Nanoparticles for Quantitative Determination of Hydrogen Peroxide. Sens. Actuators, B 2017, 251, 624-631.

(28) ChemBuddy Refractive Indices of Sucrose Solutions at $20{ }^{\circ} \mathrm{C}$, $589.29 \mathrm{Nm}$. http://www.refractometer.pl/refraction-datasheetsucrose (accessed Aug 01, 2017).

(29) Jain, P. K.; El-Sayed, M. A. Plasmonic Coupling in Noble Metal Nanostructures. Chem. Phys. Lett. 2010, 487, 153-164.

(30) Sherry, L. J.; Chang, S.-H.; Schatz, G. C.; Van Duyne, R. P.; Wiley, B. J.; Xia, Y. Localized Surface Plasmon Resonance 
Spectroscopy of Single Silver Nanocubes. Nano Lett. 2005, 5, 20342038.

(31) Galush, W. J.; Shelby, S. A.; Mulvihill, M. J.; Tao, A.; Yang, P.; Groves, J. T. A Nanocube Plasmonic Sensor for Molecular Binding on Membrane Surfaces. Nano Lett. 2009, 9, 2077-2082.

(32) Little, T. A. Method Validation Essentials, Limit of Blank, Limit of Detection, and Limit of Quantitation. BioPharm Int. 2015, 28, 4851.

(33) López-Muñoz, G. A.; Estevez, M. C.; Peláez-Gutierrez, E. C.; Homs-Corbera, A.; García-Hernandez, M. C.; Imbaud, J. I.; Lechuga, L. M. A Label-Free Nanostructured Plasmonic Biosensor Based on Blu-Ray Discs with Integrated Microfluidics for Sensitive Biodetection. Biosens. Bioelectron. 2017, 96, 260-267.

(34) Haes, A. J.; Van Duyne, R. P. A Nanoscale Optical Blosensor: Sensitivity and Selectivity of an Approach Based on the Localized Surface Plasmon Resonance Spectroscopy of Triangular Silver Nanoparticles. J. Am. Chem. Soc. 2002, 124, 10596-10604. 